Revista Investigaciones y Aplicaciones

Nucleares, 5, 84-98, 2021

Recibido: 30 de junio de 2021

Aceptado: 29 de septiembre de 2021

Publicado en línea: 23 de diciembre de 2021

Doi: https://doi.org/10.32685/2590-7468/

invapnuclear.5.2021.604

\section{(c) (i)}

Esta obra está bajo licencia internacional Creative Commons Reconocimiento 4.0.

\title{
Determinación de los niveles de referencia de dosis (DRL) para diagnóstico de baja y media complejidad en Servicios Especiales de Salud Hospital Universitario de Caldas de Colombia (SES-HUC)
}

Determination of the diagnostic dose reference level (DRL) for low and middle complexity procedures at the SES Caldas University Hospital

Estefanía Amaya Ríos ${ }^{1}$, Erika Muñoz Arango ${ }^{1}$

Citación: E. Amaya y E. Muñoz, "Determinación de los niveles de referencia de dosis (DRL) para diagnóstico de baja y media complejidad en Servicios Especiales de Salud Hospital Universitario de Caldas de Colombia (SES-HUC)”, Revista Investigaciones y Aplicaciones Nucleares, n. 5, pp. 84-98, 2021. https://doi.org/10.32685/2590-7468/invapnuclear.5.2021.604

\section{Resumen}

Objetivo: Los niveles de referencia de dosis para diagnóstico (dose reference levels - DRL) son una herramienta útil para optimizar la protección radiológica en exposiciones médicas de diagnóstico. Su determinación es un requisito para obtener la licencia de uso de equipos emisores de radiación ionizante en los servicios de imágenes diagnósticas de Colombia. El objetivo de este trabajo fue establecer los valores de DRL institucionales con diversos equipos emisores de radiación ionizante para los procedimientos de diagnósticos más comunes asociados a áreas anatómicas típicas.

Metodología: Este estudio fue realizado en dos equipos de radiología convencional, un equipo de mamografía, un equipo de tomografía computada (TC) y un equipo de fluoroscopia tipo arco en C. La muestra estadística se clasificó de acuerdo con los tipos de estudio, las áreas anatómicas de interés, tamaño y rangos de pesos de pacientes. Los tamaños de la muestra variaron según la técnica diagnóstica, siguiendo recomendaciones del documento técnico "Diagnostic reference levels in medical imaging. ICRP Publication 135". Los datos de la dosis entregada a cada paciente se obtuvieron registrando el valor de dosis estimado por el equipo de adquisición de imagen. El análisis estadístico se hizo por medio de diagramas de

${ }^{1}$ Área de Física Médica Hospitalaria, Servicios Especiales de Salud, Hospital Universitario de Caldas (SES-HUC), Manizales, Colombia.

Autor de correspondencia: Estefanía Amaya, ingenieriafisica@ses.com.co 
cajas y distribuciones de frecuencias, donde el tercer cuartil se definió como el valor de DRL para cada técnica de adquisición de imagen.

Resultados: Los valores obtenidos de DRL para radiografía convencional fueron inferiores a $15 \mathrm{dGy} . \mathrm{cm}^{2}$ para pacientes con pesos entre $50-80 \mathrm{~kg}$, e inferiores a $27 \mathrm{dGy} . \mathrm{cm}^{2}$ para pacientes de más de $80 \mathrm{~kg}$. Para mamografía, los valores de DRL a través de la dosis glandular media fueron inferiores a 2,7 mGy para proyecciones oblicuas con espesores entre 45-94 mm. Los índices de dosis en tomografía computarizada en volumen $\left(\mathrm{CTDI}_{\mathrm{vol}}\right)$ y los valores de producto dosis-longitud (DLP) fueron inferiores a 41,4 mGy y $907 \mathrm{mGy} . c \mathrm{~cm}$ respectivamente, obteniendo los mayores valores en cráneo y los menores en abdomen para pacientes entre 50 y $80 \mathrm{~kg}$. En procedimientos con arco en C, los valores obtenidos de DRL fueron iguales a 2,69 Gy.cm ${ }^{2}$ para colangiopancreatografía retrógrada endoscópica (CPRE), 2,88 Gy.cm² para histerosalpingografía (HSG) y $9,22 \mathrm{~Gy} . \mathrm{cm}^{2}$ para colon por enema.

Conclusiones: Los niveles de referencia de dosis en procedimientos diagnósticos obtenidos en este estudio para SES-HUC, han arrojado valores comparables a los publicados por otros autores en análisis similares, aplicando métodos de análisis sugeridos por organismos internacionales.

Palabras clave: niveles de referencia para diagnóstico, dosis de radiación, radiología convencional, tomografía computarizada, mamografía, fluoroscopia.

\section{Abstract}

Purpose: Diagnostic dose reference levels (DRLs) are a feasible tool for maximizing radiation protection during medical diagnostic exposures. DRL determination is a requirement for obtaining radiation ionizing operational licenses for radiodiagnostic facilities (or departments) in Colombia. The objective of this study was to determine institutional DRLs for different ionizing radiation devices used in common diagnostic procedures for typical anatomical areas. Methods: Two conventional radiology equipments, one mammography equipment, one computed tomography (CT) equipment, and a C-arm fluoroscope were investigated in this study. Statistical samples were classified in terms of the study type, anatomical area of interest, sample size, and patient weight range. The sample sizes varied with the diagnostic technique, following the recommendations of the technical document "Diagnostic reference levels in medical imaging. ICRP Publication 135". The delivered dose to each patient was determined by registering the estimated dose by the imaging acquisition device. Statistical analyses were performed using box plots and frequency distributions, with the third quartile defined as the DRL for each image acquisition technique.

Results: The DRLs for conventional radiography were lower than $15 \mathrm{dGy} . \mathrm{cm}^{2}$ for patients weighing between 50 and $80 \mathrm{~kg}$ and lower than $27 \mathrm{dGy} . \mathrm{cm}^{2}$ for patients weighing more than $80 \mathrm{~kg}$. The DRLs for mammography, based on the mean glandular dose were lower than 2.7 mGy for oblique projections with thicknesses between 45 and $94 \mathrm{~mm}$. The computed tomography volume dose index (CTDIvol) and dose-length product (DLP) were lower than 41.4 $\mathrm{mGy}$ and $907 \mathrm{mGy} . \mathrm{cm}$, respectively, where higher doses were found for the cranium and minor doses were found for the abdomen for patients weighing between 50 and $80 \mathrm{~kg}$. The DRLs for C-arm procedures were $2.69 \mathrm{~Gy} . \mathrm{cm}^{2}$ for endoscopic retrograde cholangiopancreatography procedures (CPRE), $2.88{\mathrm{~Gy} . \mathrm{cm}^{2}}^{2}$ for hysterosalpingography procedures (HSG), and $9.22 \mathrm{~Gy} . \mathrm{cm}^{2}$ for barium enema. 
Conclusions: The dose reference levels were determined for SES HUC diagnostic procedures that were comparable to published values based on similar analyses using analysis methods suggested by international organizations.

Keywords: diagnostic dose reference levels, radiation dose, radiology, computed tomography, mammography, fluoroscopy.

\section{Introducción}

D e acuerdo con el informe del Comité Científico de Naciones Unidas para el Estudio de los Efectos de las Radiaciones Atómicas (Unscear, por su sigla en inglés), la radiación generada por procedimientos médicos constituye un $19,7 \%$ de la dosis de radiación media total recibida por una persona durante un año. De esta forma, los servicios de atención médica deben utilizar la radiación ionizante de forma que se pueda lograr el máximo beneficio posible con el mínimo riesgo para el paciente [1].

La determinación de los niveles de referencia de dosis (DRL, por su sigla en inglés) se utiliza para ayudar a optimizar la protección radiológica en las exposiciones de pacientes sometidos a procedimientos de diagnóstico e intervencionismo; estos indican si, en condiciones de rutina, la cantidad de radiación utilizada para un procedimiento específico es inusualmente alta o baja para dicho estudio, considerando que la calidad de la imagen no se vea comprometida. Estos valores no son límites de dosis y no se aplican para un individuo, sino que se emplean para un grupo poblacional [2], [3].

La definición de los DRL se hace evaluando diversas magnitudes de dosis de acuerdo con la modalidad de imagen empleada. Dichas magnitudes de dosis son: producto dosis-longitud (dose-length-product - DLP) en tomografía, producto dosis-área (dose-area-product - DAP) en radiografía convencional y fluoroscopia, índice de dosis en tomografía computarizada en volumen (volume computed tomography dose index - $\mathrm{CTDI}_{\mathrm{vol}}$ ) y dosis glandular media (average-glandular-dose - AGD) para equipos de mamografía. Estas se correlacionan con la energía impartida al volumen de interés y no con las dosis absorbidas en los tejidos y órganos del paciente [2].

Para la definición de los DRL, diversas metodologías suelen ser aplicadas en el ámbito de las imágenes diagnósticas. En el estudio de Vawda et al. [4], se establecieron los niveles de referencia de diagnóstico para tomografía computarizada (TC), mediante la determinación de los valores medios de CTDI $I_{\mathrm{vol}}$ y DLP, y la clasificación de los pacientes por rangos de edad. En el estudio de Benmessaoud et al. [5], se trabajaron los DRL con el criterio del percentil 75 de las

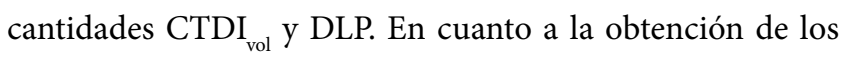
valores de dosis, según el documento técnico "Diagnostic reference levels in medical imaging, ICRP Publication 135”, los niveles de referencia para diagnóstico pueden obtenerse ya sea por mediciones dosimétricas directas (cuando se cuenta con los equipos de dosimetría) o por análisis de datos desde el propio equipo [2].

En Colombia, el uso de equipos de obtención de imágenes diagnósticas emisores de rayos $\mathrm{X}$ se rige por la Resolución 482 de 2018 del Ministerio de Salud y Protección Social. Dentro de los requisitos de licenciamiento está la definición institucional de los DRL para los procedimientos más comunes con cada equipo [3].

En este estudio se han estimado los valores típicos de dosis de radiación impartida en los procedimientos médicos de diagnóstico más comunes que se adelantan en Servicios Especiales de Salud Hospital Universitario de Caldas (SESHUC), con técnicas que utilizan equipos generadores de rayos X de baja-media energía. Se han aplicado principalmente las recomendaciones del documento técnico "ICRP 135" [2]. En este estudio, se definió como DRL el tercer cuartil del diagrama de cajas obtenido para cada grupo evaluado.

\section{Metodología}

\subsection{Definición de la muestra estadística de pacientes}

Los datos fueron recolectados durante todo el primer semestre e inicios del segundo semestre de 2020.

La clasificación de la muestra poblacional se definió para los procedimientos de radiodiagnóstico más comunes dentro de las técnicas que se llevan a cabo en la institución. En la definición del tamaño de la muestra (n), descrito en la tabla 1, fueron consideradas las recomendaciones del documento técnico ICRP 135 [2] para los diferentes tipos de imágenes diagnósticas. 


\subsubsection{Características de la muestra para radiología}

\section{convencional}

Para radiología convencional, se seleccionaron los estudios más comunes en la institución: radiografía de tórax, radiografía de pelvis y radiografía de abdomen. La clasificación de los grupos se hizo, además del tipo de estudio, por tamaño de paciente según la denominación "paciente grande" y "paciente mediano", y no hubo clasificación de grupos por género (hombre o mujer). La definición de "paciente grande" o "paciente mediano" está asociada al espesor del paciente (especificado en el manual de usuario del equipo) y corresponden a rangos de pesos entre 50 y $80 \mathrm{~kg}$ para paciente mediano, y mayor a 80 kg para paciente grande (se excluyeron los pesos atípicos para paciente mediano $<50 \mathrm{~kg}$ y para paciente grande $>90 \mathrm{~kg}$ ).

Los rangos de espesores para paciente grande y mediano se presentan en la tabla 1, así como la edad promedio, el rango de edades y el tamaño de la muestra, con discriminación del número de hombres y mujeres. Para radiografía de tórax no se contó con una muestra suficiente para el análisis de adulto grande (la muestra era inferior a $n=10$ ). Para el análisis de neonatos, específicamente, el rango de pesos se presenta en la tabla 1 .

\subsubsection{Características de la muestra para mamografía}

En el estudio fueron consideradas mamas de dos tipos, según la clasificación del Colegio Americano de Radiología (ACR, por su sigla en inglés):

a. Mamas con parénquima mamario con sectores dispersos de densidad fibroglandular (ACR B) con 25-50 \% glándula, siendo esta la de mayor prevalencia en la muestra.

b. Mamas con parénquima mamario de tipo heterogéneamente denso (ACR C) con 50-75 \% glándula [6].
En el estudio se descartaron mamas con prótesis o que correspondieran a otra clasificación por densidad, como las mamas con parénquima de tipo predominante adiposo (ACR A) $<25 \%$ de glándula, y mamas con parénquima mamario de tipo extremadamente denso (ACR D) > $75 \%$ de glándula [6].

Para el análisis de los valores de referencia se evaluaron las cuatro proyecciones típicas de adquisición de imagen y estas están asociadas a diferentes espesores, según la proyección, los cuales se presentan en la tabla 1, así como la edad promedio, el rango de edades y el valor de la muestra.

\subsubsection{Características de la muestra para tomografía computarizada}

Para tomografía computarizada, se seleccionaron los estudios más comunes en la institución: tomografía de tórax, tomografía de abdomen y tomografía de cráneo. La clasificación de los grupos se realizó, además, de acuerdo con la región anatómica que corresponde a un protocolo específico de adquisición en el equipo. Para el estudio fueron seleccionados solo pacientes medianos con peso en el rango entre 50 y $80 \mathrm{~kg}$. En la tabla 1 se presentan los rangos de edades y el tamaño de la muestra, discriminando femenino y masculino para las tres áreas anatómicas.

\subsubsection{Características de la muestra para procedimientos guiados por fluoroscopia con arco en C}

Para estos estudios se presenta en la tabla 1 el tamaño de la muestra, discriminando entre masculino y femenino para el colon por enema y CPRE, así como los rangos de edades. La técnica de HSG solo se realiza en mujeres en edad fértil. No se hicieron clasificaciones por peso, talla u otros parámetros debido a la gran variabilidad de parámetros en estas técnicas.

Tabla 1. Clasificación de la muestra estadística siguiendo las recomendaciones del ICRP 135 [2]

\begin{tabular}{|c|c|c|c|}
\hline Estudio & Dimensión anatómica & $\begin{array}{c}\text { Número de pacientes (n) } \\
\text { Femenino (F), Masculino (M) }\end{array}$ & Edad promedio y rango (años) \\
\hline Radiografía de tórax & $\begin{array}{c}\text { Adulto mediano: } \\
22 \mathrm{~cm} \leq \text { espesor de tórax } \leq 27 \mathrm{~cm} \\
50 \mathrm{~kg} \leq \text { peso } \leq 80 \mathrm{~kg}\end{array}$ & $\begin{array}{c}\mathrm{n}=29 \\
\mathrm{~F}: 17, \mathrm{M}: 12\end{array}$ & $\begin{array}{c}61 \\
{[21-80]}\end{array}$ \\
\hline \multirow{4}{*}{ Radiografía de pelvis } & \multirow{2}{*}{$\begin{array}{c}\text { Adulto mediano: } \\
22 \mathrm{~cm} \leq \text { espesor de tórax } \leq 27 \mathrm{~cm} \\
50 \mathrm{~kg} \leq \text { peso } \leq 80 \mathrm{~kg}\end{array}$} & $\begin{array}{c}\mathrm{n}=24 \\
\mathrm{~F}: 13, \mathrm{M}: 11\end{array}$ & $\begin{array}{c}49 \\
{[18-80]} \\
\end{array}$ \\
\hline & & $\begin{array}{c}\mathrm{n}=13 \\
\mathrm{~F}: 6, \mathrm{M}: 7\end{array}$ & $\begin{array}{c}48 \\
{[20-76]}\end{array}$ \\
\hline & Adulto grande: & $\mathrm{n}=13$ & 64 \\
\hline & espesor de pelvis $>27 \mathrm{~cm}$; peso $>80 \mathrm{~kg}$ & $\mathrm{~F}: 6, \mathrm{M}: 7$ & [47-81] \\
\hline \multirow[t]{2}{*}{ Radiografía de abdomen } & $\begin{array}{c}\text { Adulto mediano: } \\
22 \mathrm{~cm} \leq \text { espesor de tórax } \leq 27 \mathrm{~cm} \\
50 \mathrm{~kg} \leq \text { peso } \leq 80 \mathrm{~kg}\end{array}$ & $\begin{array}{c}\mathrm{n}=27 \\
\mathrm{~F}: 18, \mathrm{M}: 9\end{array}$ & $\begin{array}{c}55,5 \\
{[23-88]}\end{array}$ \\
\hline & $\begin{array}{c}\text { Adulto grande: } \\
\text { espesor }>27 \mathrm{~cm} \text {; peso }>80 \mathrm{~kg}\end{array}$ & $\begin{array}{c}\mathrm{n}=11 \\
\mathrm{~F}: 4, \mathrm{M}: 7\end{array}$ & $\begin{array}{c}65,5 \\
{[47-84]}\end{array}$ \\
\hline
\end{tabular}




\begin{tabular}{|c|c|c|c|}
\hline Estudio & Dimensión anatómica & $\begin{array}{c}\text { Número de pacientes (n) } \\
\text { Femenino (F), Masculino (M) }\end{array}$ & Edad promedio y rango (años) \\
\hline & $\begin{array}{l}\text { Neonato mediano: } \\
2 \mathrm{~kg}<\text { peso }<5 \mathrm{~kg}\end{array}$ & $\mathrm{n}=24$ & 0 \\
\hline Radiografía de tórax portátil & $\begin{array}{c}\text { Adulto mediano: } \\
22 \mathrm{~cm} \leq \text { espesor de tórax } \leq 27 \mathrm{~cm} \\
50 \mathrm{~kg} \leq \text { peso } \leq 80 \mathrm{~kg}\end{array}$ & $\begin{array}{c}\mathrm{n}=30 \\
\mathrm{~F}: 10, \mathrm{M}: 20\end{array}$ & $\begin{array}{c}56,5 \\
{[32-81]}\end{array}$ \\
\hline Mamografía & $\begin{array}{c}\text { Espesor de la mama }(\mathrm{mm}) \\
40-74(\mathrm{RCC}) \\
45-79(\mathrm{RMLO}) \\
35-102(\mathrm{LCC}) \\
46-94(\mathrm{LMLO}) \\
\end{array}$ & $\begin{array}{c}\mathrm{n}=50 \\
\mathrm{~F}: 50, \mathrm{M}: 0\end{array}$ & $\begin{array}{c}60 \\
{[50-70]}\end{array}$ \\
\hline Tomografía computarizada & Diámetro equivalente de agua $(\mathrm{cm})$ & & \\
\hline TC de cráneo & ( & $\begin{array}{c}\mathrm{n}=30 \\
\mathrm{~F}: 22, \mathrm{M}: 8\end{array}$ & $\begin{array}{c}53,5 \\
{[25-82]} \\
\end{array}$ \\
\hline TC de tórax & 32 & $\begin{array}{c}\mathrm{n}=30 \\
\mathrm{~F}: 11, \mathrm{M}: 19\end{array}$ & $\begin{array}{c}60 \\
{[30-90]}\end{array}$ \\
\hline TC abdomen & 32 & $\begin{array}{c}n=30 \\
F: 17, M: 13\end{array}$ & $\begin{array}{c}58 \\
{[25-91]}\end{array}$ \\
\hline Fluoroscopia con $\operatorname{arco}$ en $\mathrm{C}$ & & & \\
\hline Colon por enema & $\mathrm{NA}$ & $\begin{array}{c}\mathrm{n}=20 \\
\mathrm{~F}: 11, \mathrm{M}: 9\end{array}$ & $\begin{array}{c}49 \\
{[20-78]}\end{array}$ \\
\hline HSG & $\mathrm{NA}$ & $\begin{array}{c}\mathrm{n}=20 \\
\mathrm{~F}: 20\end{array}$ & $\begin{array}{c}30,5 \\
{[25-36]}\end{array}$ \\
\hline CPRE & NA & $\begin{array}{c}\mathrm{n}=30 \\
\mathrm{~F}: 11, \mathrm{M}: 19\end{array}$ & $\begin{array}{c}53,5 \\
{[28-79]}\end{array}$ \\
\hline
\end{tabular}

\subsection{Modalidades de imagen}

Los valores de dosis entregada por estudio se obtuvieron de la dosis estimada por el equipo generador de imagen, a los que se les realizó, con anterioridad a la toma de datos, un control de calidad que cumple con los requisitos de la legislación nacional, Resolución 482 de 2018. [7].

El estudio se hizo para los siguientes equipos: un equipo de rayos X convencional fijo General Electric, modelo OPTIMA XR646; un equipo de rayos X convencional portátil General Electric, modelo OPTIMA XR220; un mamógrafo General Electric, modelo Senographe Essential; un tomógrafo PHILIPS, modelo Ingenuity CT, y un equipo de rayos $\mathrm{X}$ tipo arco en C General Electric, modelo OEC Elite.

La evaluación de los grupos fue clasificada según el equipo y el $\mathrm{kVp}$ utilizado y dimensiones anatómicas basadas en protocolos según se presenta en las tablas 1 y 2 . También se hicieron análisis específicos según las proyecciones tomadas durante el procedimiento a evaluar, como se detalla en la tabla 2. Todas las imágenes incluidas en el estudio cumplen con criterios de adecuada calidad de imagen diagnóstica según el consenso de los especialistas de la institución, la cual se obtiene principalmente aplicando los protocolos previamente cargados en los equipos.

\subsubsection{Radiología convencional}

Los estudios más realizados en la institución con el equipo digital de radiología convencional fijo son: radiografía de tórax con proyecciones postero-anterior (PA) y lateral
(LAT) y radiografía de pelvis y abdomen en su proyección antero-posterior (AP). Para el equipo de rayos X portátil, el estudio más común es la radiografía de tórax en pacientes adultos y neonatales, con una sola proyección AP.

La clasificación de la muestra se hizo según el valor de $\mathrm{kVp}$ que el equipo tiene como protocolo predeterminado para cada procedimiento, y tamaño del paciente (mediano o grande). Para los estudios de rayos $\mathrm{X}$ convencional, la magnitud de dosis analizada fue el DAP estimado por el equipo.

\subsubsection{Mamografía}

El estudio más común adelantado en el mamógrafo requiere 4 proyecciones de la mama: cráneo caudal derecha (right-cranio-caudal - RCC), oblicua medio lateral derecha (right-mediolateral-oblique - RMLO), cráneo caudal izquierda (left-cranio-caudal - LCC) y oblicua medio lateral izquierda (left-mediolateral-oblique - LMLO). La clasificación de la muestra se hizo por el tipo de proyección, donde a su vez se considera la variación en el espesor de la mama (con el kVp en un intervalo entre 27 y $31 \mathrm{kVp}$ ). La magnitud de dosis analizada fue la AGD.

\subsubsection{Tomografía computarizada}

Para el análisis fueron elegidos los estudios correspondientes a cráneo, tórax y abdomen para paciente adulto mediano, siendo esta la base de la clasificación de los grupos para el estudio. Otros parámetros como longitud del barrido, campo 
de visión (field of view -FOV) y espesor de corte están directamente relacionados con el área anatómica y, a su vez, con el protocolo del equipo así:

1 Longitud del scan promedio de la muestra por protocolo: cráneo $162 \mathrm{~mm}$; tórax 312,3 mm; abdomen 435,3 mm.

» FOV promedio de la muestra por área anatómica: cráneo 225,3 mm; tórax 320,3 mm; abdomen $358 \mathrm{~mm}$

» Espesores de corte promedio de acuerdo con el área anatómica. Definido para cada protocolo: cráneo 1,50 mm; tórax 1,00 $\mathrm{mm}$, abdomen $2,00 \mathrm{~mm}$.

Todos los estudios analizados fueron adquiridos con $120 \mathrm{kVp}$, parámetro predeterminado en el equipo en cada uno de estos protocolos. En la tabla 2 se presenta el intervalo de mAs correspondiente para cada área anatómica y adquisición (topograma y adquisición helicoidal). Para el área anatómica tórax, cada paciente tiene dos adquisiciones helicoidales en los rangos de $\mathrm{mAs}$ presentados. El equipo aplica modulación automática de la corriente del tubo, adaptando los mAs del tubo con el objetivo de optimizar las dosis manteniendo la calidad de imagen.

Las magnitudes de dosis analizadas en los procedimientos de tomografía fueron el CTDI ${ }_{\text {vol }}$ y el DLP de todo el examen, incluyendo el topograma, estimados por el equipo.

\subsubsection{Procedimientos guiados por fluoroscopia con arco}

en $C$

Para procedimientos guiados por fluoroscopia se eligieron tres estudios frecuentes en la institución, empleando el arco en C: colon por enema, histerosalpingografía (HSG) y colangiopancreatografía retrógrada endoscópica (CPRE), y la magnitud de dosis evaluada fue el DAP.

Debido a que en estos procedimientos varía el tiempo de irradiación por la complejidad de cada estudio, al igual que el kVp por el espesor del paciente y por la posición del arco, se definió que la manera más adecuada de clasificación de los grupos se hiciera por estudio.

\subsection{Análisis estadístico de datos}

Se realizaron diagramas de cajas (box-plot) con los datos adquiridos por estudio, con el fin de analizar las magnitudes dosimétricas por medio de su distribución en cuartiles. Los datos observados fueron los siguientes: la mediana, el primer cuartil, el rango intercuartílico y el tercer cuartil [8]. El tercer cuartil del diagrama de caja se definió en este estudio como el DRL según las clasificaciones propuestas.

Tabla 2. Estudios diagnósticos más comunes en SES-HUC por equipo emisor de rayos X, con las proyecciones que requieren, clasificación por tamaño del paciente, clasificación por kVp utilizado, rango de mAs utilizado, magnitud dosimétrica

\begin{tabular}{|c|c|c|c|c|c|c|}
\hline Equipo emisor de RX & Estudio & Proyección & Dimensión anatómica & $\mathbf{k V p}$ & Rango de mAs & $\begin{array}{c}\text { Magnitud y unidad } \\
\text { dosimétrica }\end{array}$ \\
\hline \multirow{6}{*}{ Rayos X fijo } & Radiografía de tórax & $\begin{array}{c}\text { PA } \\
\text { LAT }\end{array}$ & $\begin{array}{c}22 \mathrm{~cm} \leq \text { espesor de } \\
\text { tórax } \leq 27 \mathrm{~cm}\end{array}$ & 120 & $1,49-9,15$ & \multirow{6}{*}{$\begin{array}{c}\text { DAP } \\
\left(\mathrm{dGy} \cdot \mathrm{cm}^{2}\right)\end{array}$} \\
\hline & \multirow{3}{*}{ Radiografía de pelvis } & \multirow{3}{*}{ AP } & $22 \mathrm{~cm} \leq$ espesor de & 80 & $4,65-17,87$ & \\
\hline & & & tórax $\leq 27 \mathrm{~cm}$ & 85 & $11,64-12,39$ & \\
\hline & & & espesor $>27 \mathrm{~cm}$ & 80 & $15,06-31,86$ & \\
\hline & \multirow{2}{*}{$\begin{array}{c}\text { Radiografía de } \\
\text { abdomen }\end{array}$} & \multirow[t]{2}{*}{ AP } & $\begin{array}{c}22 \mathrm{~cm} \leq \text { espesor de } \\
\text { tórax } \leq 27 \mathrm{~cm}\end{array}$ & 80 & $1,88-77,74$ & \\
\hline & & & espesor $>27 \mathrm{~cm}$ & 85 & $13,49-99,51$ & \\
\hline \multirow[b]{2}{*}{ Rayos X portátil } & \multirow[b]{2}{*}{ Radiografía de tórax } & \multirow[b]{2}{*}{$\mathrm{AP}$} & $2 \mathrm{~kg}<$ peso $<5 \mathrm{~kg}$ & 65 & $0,66-0,85$ & \multirow[b]{2}{*}{$\begin{array}{c}\text { DAP } \\
\left(\mathrm{dGy} \cdot \mathrm{cm}^{2}\right)\end{array}$} \\
\hline & & & $\begin{array}{l}22 \mathrm{~cm} \leq \text { espesor de } \\
\text { tórax } \leq 27 \mathrm{~cm}\end{array}$ & 95 & $1,62-1,63$ & \\
\hline \multirow{5}{*}{ Mamógrafo } & \multirow{5}{*}{ Mamografía } & & $\begin{array}{c}\text { Espesor de la mama } \\
(\mathrm{mm})\end{array}$ & & & \multirow{5}{*}{$\begin{array}{l}\text { AGD } \\
(\mathrm{mGy})\end{array}$} \\
\hline & & RCC & $40-74$ & $27-31$ & $45-206,2$ & \\
\hline & & RMLO & $45-79$ & $28-31$ & $45-222,80$ & \\
\hline & & LCC & $35-102$ & $27-31$ & $45-324$ & \\
\hline & & LMLO & $46-94$ & $28-31$ & $40-324$ & \\
\hline \multirow{8}{*}{ Tomógrafo } & $\begin{array}{c}\text { Tomografía } \\
\text { computarizada }\end{array}$ & & $\begin{array}{c}\text { Diámetro equivalente } \\
\text { de agua (cm) }\end{array}$ & & & \multirow{8}{*}{$\begin{array}{c}\text { CTDI }_{\text {vol }} \\
(\mathrm{mGy}) \\
\text { DLP } \\
\text { (mGy.cm) }\end{array}$} \\
\hline & \multirow{2}{*}{ TC de cráneo } & Topograma & 32 & 120 & - & \\
\hline & & Helicoidal & 16 & 120 & 320 & \\
\hline & \multirow{3}{*}{ TC de tórax } & Topograma & 32 & 120 & - & \\
\hline & & Helicoidal & 32 & 120 & $81-267$ & \\
\hline & & Helicoidal & 32 & 120 & $59-184$ & \\
\hline & \multirow{2}{*}{ TC de abdomen } & Topograma & 32 & 120 & - & \\
\hline & & Helicoidal & 32 & 120 & $60-214$ & \\
\hline \multirow{3}{*}{ Arco en C } & Colon por enema & Incidencia variable & NA & $78-92$ & & \multirow{3}{*}{$\begin{array}{c}\text { DAP } \\
\left(\mathrm{Gy} \cdot \mathrm{cm}^{2}\right)\end{array}$} \\
\hline & HSG & AP & $\mathrm{NA}$ & $78-82$ & & \\
\hline & CPRE & LAT & NA & $79-88$ & & \\
\hline
\end{tabular}




\section{Resultados y discusión}

De acuerdo con lo publicado por diferentes autores, la elección del tercer cuartil como nivel de referencia permite una evaluación más conservadora de las dosis estimadas [7], [10], [11], frente a la recomendación del ICRP 35 [2] y a lo realizado por ejemplo en el estudio de Serra et al. [9], donde se evalúa la mediana como parámetro de referencia para establecer

(a)

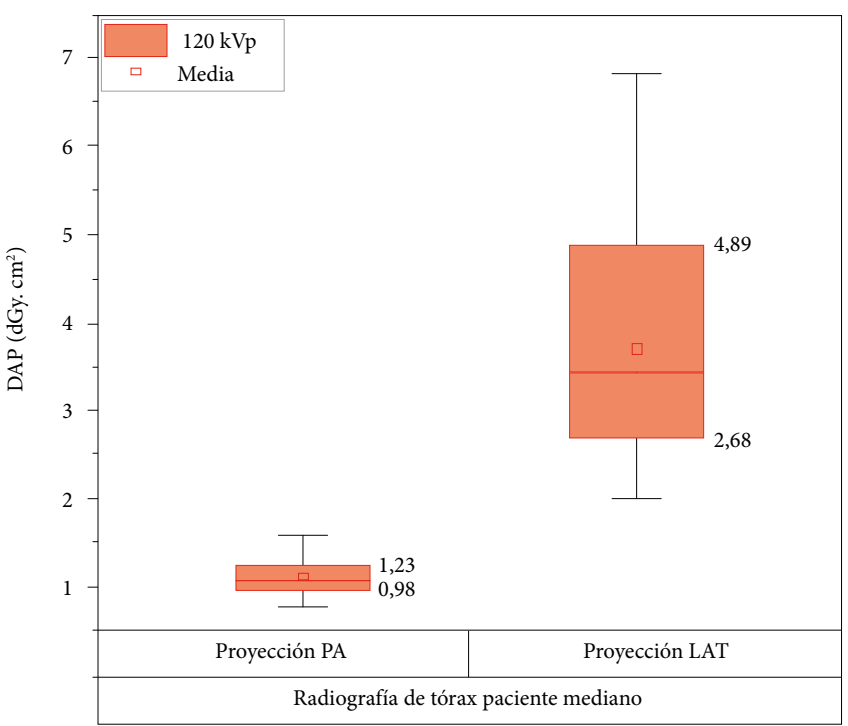

los DRL. A continuación se presentan los valores de referencia obtenidos a partir del tercer cuartil del diagrama de cajas para todas las técnicas diagnósticas consideradas.

\subsection{Nivel de referencia de dosis en radiología convencional fija}

En las figuras 1a, $1 \mathrm{~b}$ y $1 \mathrm{c}$, se muestran los diagramas de cajas para rayos $\mathrm{X}$ convencional fijo.

(b)

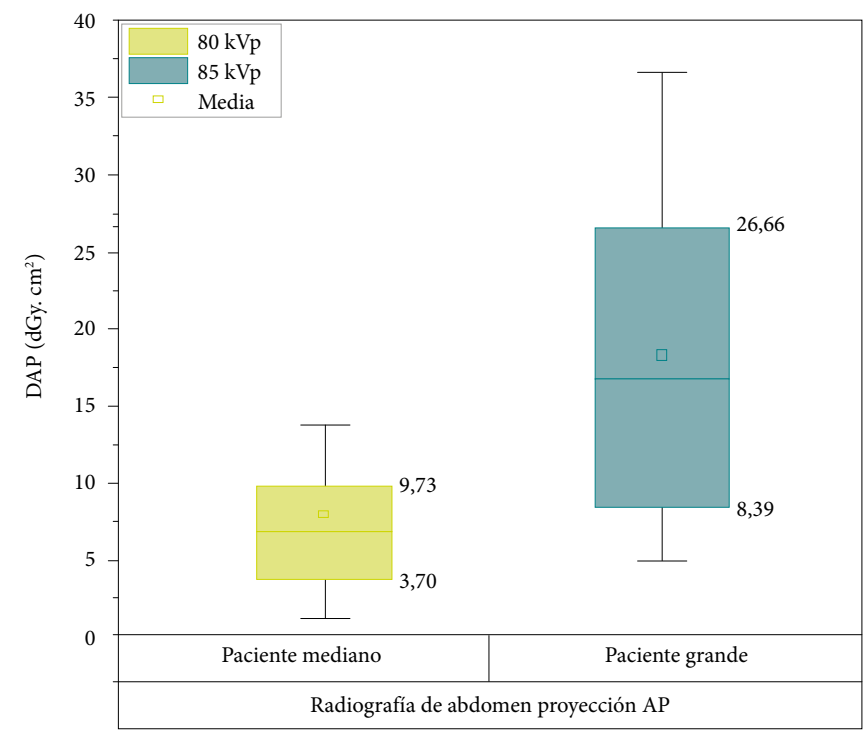

(c)

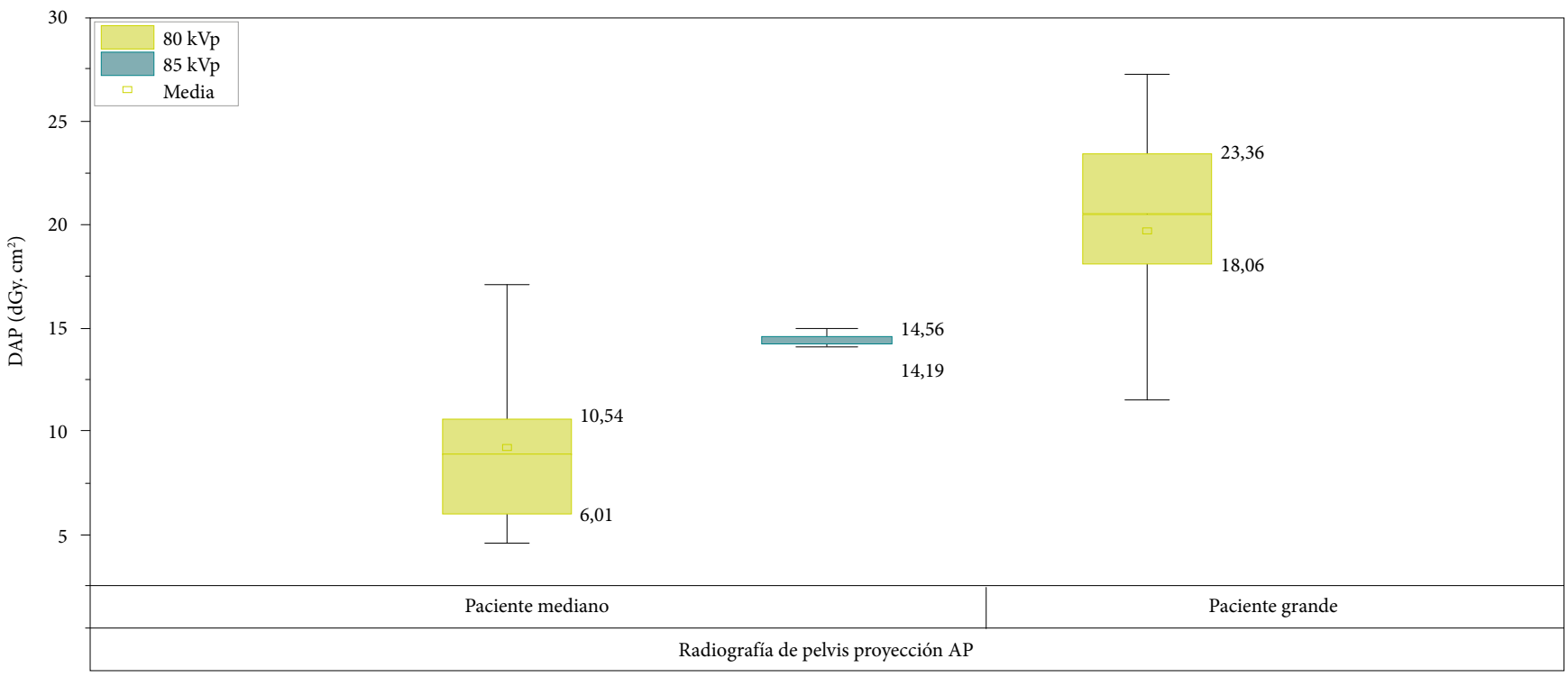

Figura 1. Gráfico de cajas del DAP

a) radiografía convencional de tórax paciente mediano $(50 \mathrm{k} \leq$ peso $\leq 80 \mathrm{~kg})$; b) radiografía convencional de abdomen paciente mediano $(50 \mathrm{~kg} \leq \mathrm{peso} \leq 80 \mathrm{~kg})$ y paciente grande (peso $>80 \mathrm{~kg}$ ); c) radiografía convencional de pelvis paciente mediano $(50 \mathrm{~kg} \leq$ peso $\leq 80 \mathrm{~kg}$ ) y paciente grande (peso $>80 \mathrm{~kg})$. 
a

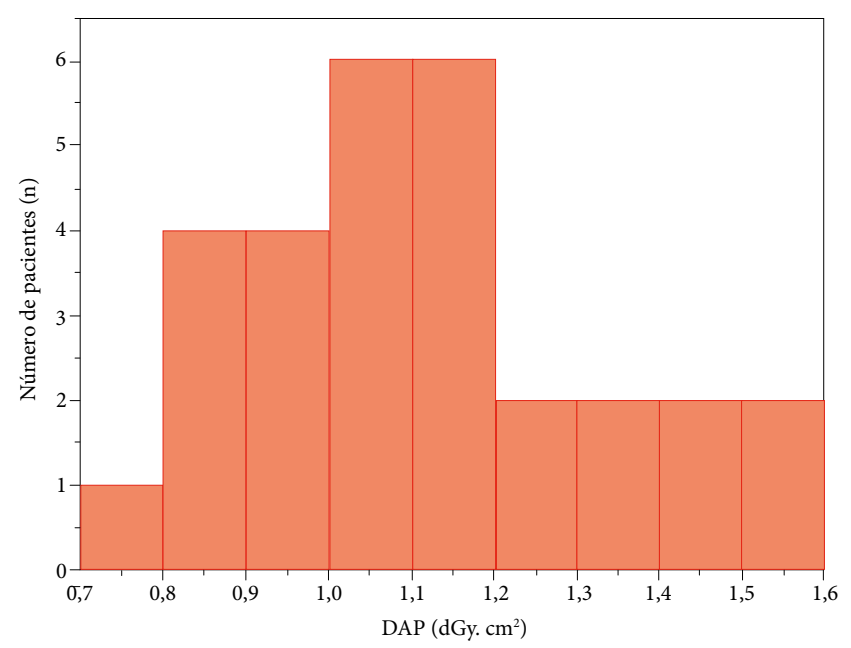

(b)

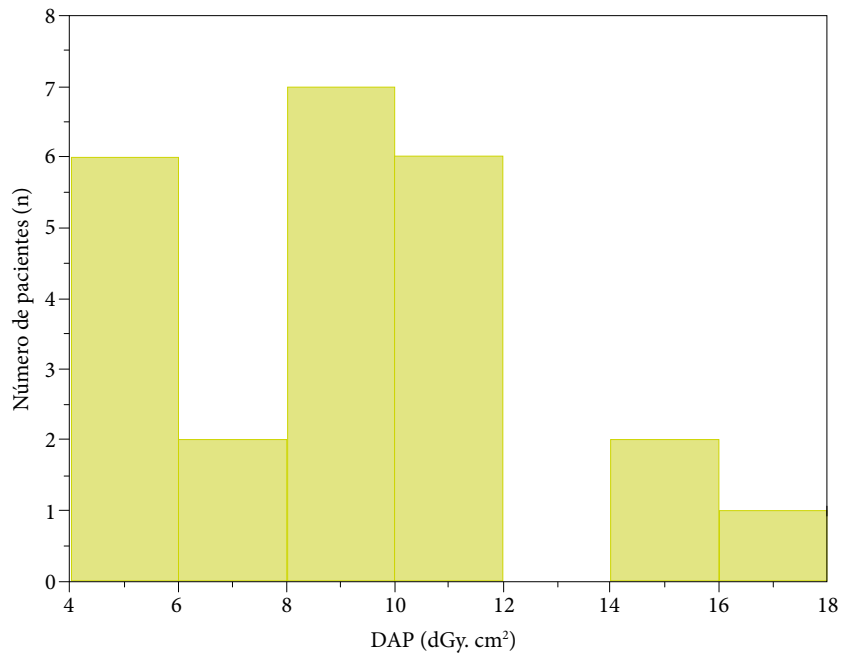

(c)

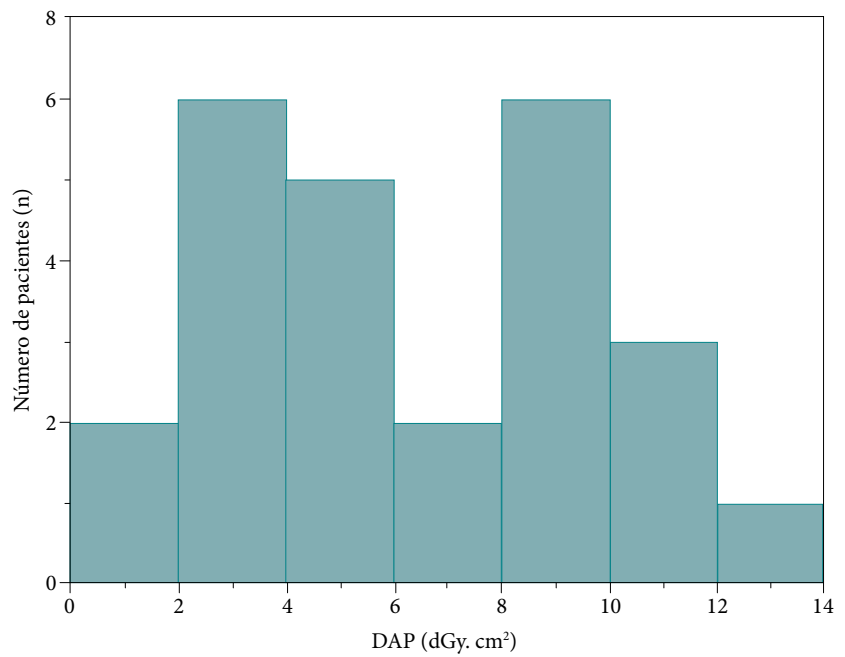

Figura 2. Diagrama de frecuencias del DAP

a) rayos $\mathrm{X}$ de tórax (PA) paciente mediano $120 \mathrm{kV}$; b) rayos $\mathrm{X}$ de pelvis (AP) paciente mediano $80 \mathrm{kV}$; c) rayos $\mathrm{X}$ de abdomen (AP) paciente mediano $80 \mathrm{kV}$.

Tabla 3. DRL para los procedimientos de radiografía de tórax, pelvis y abdomen

\begin{tabular}{|c|c|c|c|c|}
\hline Estudio & Proyección y kVp & $\begin{array}{c}\text { DRL } \\
\text { Este estudio } \\
\text { (dGy.cm²) } \\
\end{array}$ & $\begin{array}{c}\text { DRL } \\
\begin{array}{c}\text { Almén et al. [10] } \\
\text { (dGy.cm²) }\end{array}\end{array}$ & $\begin{array}{c}\text { DRL } \\
\text { NDRL [11] } \\
\text { (dGy.cm²) } \\
\end{array}$ \\
\hline \multirow{2}{*}{$\begin{array}{l}\text { Radiografía tórax } \\
\text { (paciente mediano) }\end{array}$} & PA (120 kVp) & 1,23 & 0,97 & $1,0(88 \mathrm{kVp})$ \\
\hline & LAT $(120 \mathrm{kVp})$ & 4,89 & ND & ND \\
\hline Radiografía pelvis & $\mathrm{AP}(80 \mathrm{kVp})$ & 10,54 & \multirow{2}{*}{6,46} & \multirow{3}{*}{$22(89 \mathrm{kVp})$} \\
\hline (paciente mediano) & $\mathrm{AP}(85 \mathrm{kVp})$ & 14,56 & & \\
\hline $\begin{array}{l}\text { Radiografía pelvis } \\
\text { (paciente grande) }\end{array}$ & $\mathrm{AP}(80 \mathrm{kVp})$ & 23,36 & ND & \\
\hline $\begin{array}{c}\text { Radiografía abdomen } \\
\text { (paciente mediano) }\end{array}$ & $\mathrm{AP}(80 \mathrm{kVp})$ & 9,73 & 15,4 & \multirow[b]{2}{*}{$25(76 \mathrm{kVp})$} \\
\hline $\begin{array}{c}\text { Radiografía abdomen } \\
\text { (paciente grande) }\end{array}$ & AP (85 kVp) & 26,66 & ND & \\
\hline
\end{tabular}

ND: no disponible en la publicación. 
En las figuras $2 \mathrm{a}, 2 \mathrm{~b}$ y $2 \mathrm{c}$ se muestran los diagramas de frecuencias para rayos $\mathrm{X}$ convencional, correspondientes a paciente mediano para áreas anatómicas, tórax, pelvis y abdomen. Para estos se descartaron los valores atípicos de dosis obtenidos.

Los DRL obtenidos para radiografía convencional en pacientes adultos, a partir de las figuras $1 \mathrm{a}, 1 \mathrm{~b}$ y $1 \mathrm{c}$, se muestran en la tabla 3 y se comparan con los publicados por otros autores. Dichos valores pueden observarse también en el correspondiente diagrama de frecuencias en las figuras $2 \mathrm{a}, 2 \mathrm{~b}$ y $2 c$, donde se presenta la relación del número de pacientes para los diferentes rangos de dosis obtenidos por área anatómica en paciente mediano.

Comparando los resultados obtenidos por Almén et al. [10], donde se definieron los niveles de referencia regionales a partir del tercer cuartil de una serie de estudios recolectados en 29 hospitales de cuatro países, se observa que la diferencia de los valores de DAP (DRL) obtenidos para pelvis y abdomen en pacientes medianos (entre 50-70 kg [10]) es inferior a 8,1 dGy.cm² entre ambos estudios, y para tórax en la proyección postero-anterior la diferencia es inferior a $0,3 \mathrm{dGy} \cdot \mathrm{cm}^{2}$. Las diferencias obtenidas pueden deberse a la muestra estadística y la dispersión de los datos del estudio en comparación.

Por otro lado, en comparación con los niveles de referencia nacionales del documento "National Diagnostic Reference Levels" [11] del Reino Unido, la diferencia en los valores de DAP para tórax, pelvis y abdomen son inferiores a 15,27 dGy.cm ${ }^{2}$ para paciente mediano e inferiores a $1,66 \mathrm{dGy} . \mathrm{cm}^{2}$ para paciente grande, en comparación con los valores de este
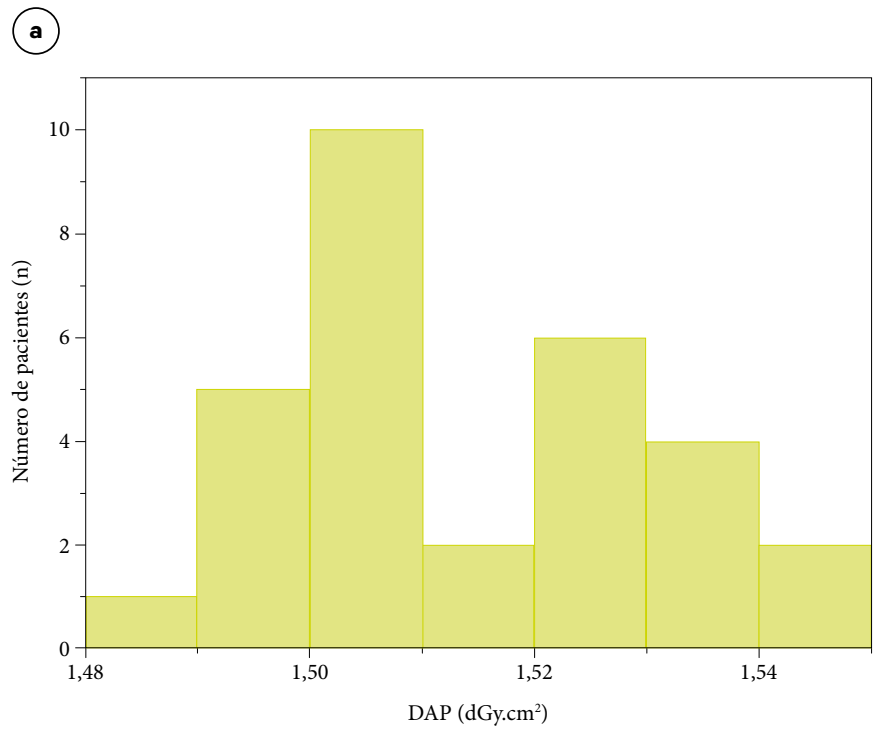

estudio. Vale la pena resaltar que en el estudio de NRDL de Reino Unido, no se realizó clasificación por peso, sino que se evaluó peso promedio, lo que presentó una dispersión considerable en los tamaños de los pacientes (entre 16-114 kg) así como en los valores de $\mathrm{kVp}$ (entre 60-125 kVp).

\subsection{Nivel de referencia de dosis en radiología convencional portátil}

En la figura 3 se muestra el diagrama de cajas para rayos $\mathrm{X}$ portátil.

En la figura 4, se presentan los diagramas de frecuencias del DAP para rayos $\mathrm{X}$ portátil, adulto mediano y neonatos, donde fueron discriminados los valores atípicos.

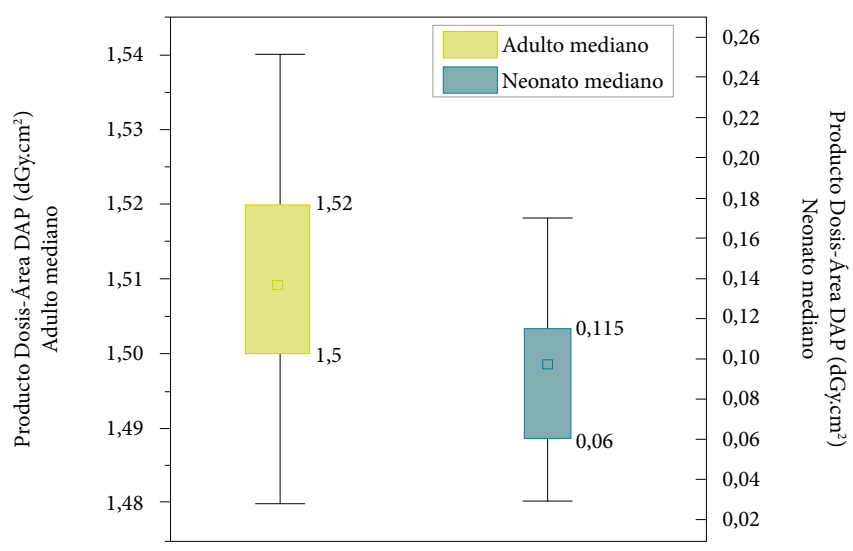

Figura 3. Gráfico de cajas de DAP para radiografía de tórax con equipo portátil para pacientes adultos medianos $(50 \mathrm{~kg}$ peso $80 \mathrm{~kg})$ y pacientes neonatales $(2 \mathrm{~kg} \leq$ peso $\leq 5 \mathrm{~kg})$

(b)

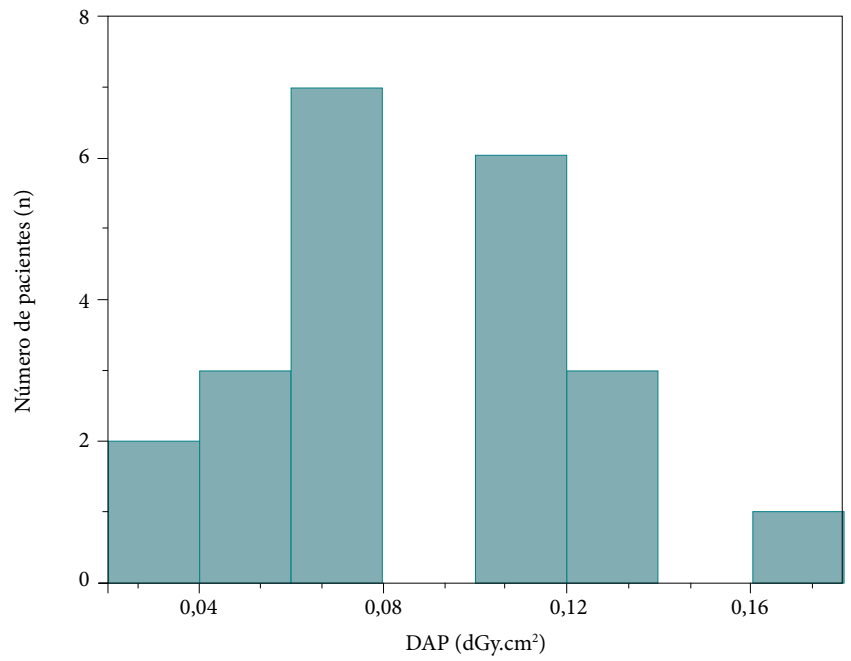

Figura 4. Diagrama de frecuencias del DAP

a) rayos $\mathrm{X}$ de tórax portátil adulto mediano; b) rayos $\mathrm{X}$ de tórax portátil neonatos. 
El DRL obtenido a partir de los DAP del diagrama de cajas fue de 1,52 dGy.cm² para pacientes adultos, alcanzando un valor similar al del documento "National Diagnostic Reference Levels" [11] del Reino Unido, donde el DAP fue igual a 1,5 dGy.cm² para radiografías de tórax en la proyección AP. Para pacientes neonatos medianos, el DAP en este estudio fue igual a 0,115 dGy.cm². Los DAP pueden observarse también en el correspondiente diagrama de frecuencias en las figuras $4 \mathrm{a} y$ $4 \mathrm{~b}$, donde se ilustra la relación del número de pacientes para los diferentes rangos de dosis para paciente adulto y neonato.

\subsection{Nivel de referencia de dosis en mamografía}

En la figura 5 se muestra el diagrama de cajas para estudios de mamografía y en la figura 6, se presentan los diagramas de frecuencias de la AGD de todas las proyecciones y espesores.

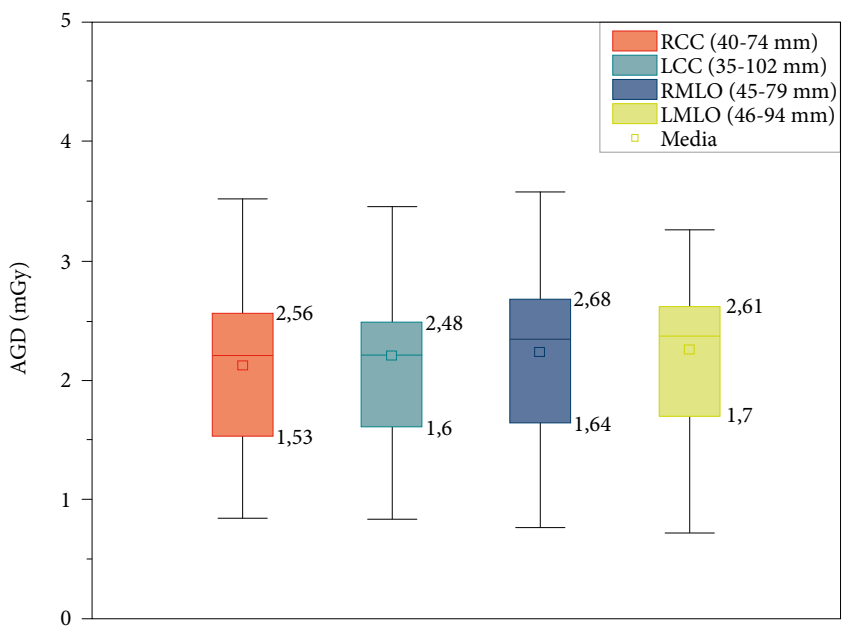

Figura 5. Gráfico de cajas de AGD para estudios de mamografía

Los DRL obtenidos por proyección fueron: 2,56 mGy para la proyección RCC (40-74 mm de espesor), 2,48 mGy para LCC (35-102 $\mathrm{mm}$ de espesor), 2,68 mGy para RMLO (45-79 mm de espesor) y 2,61 mGy para LMLO (46-94 mm de espesor), con un valor promedio de 2,64 mGy entre proyecciones oblicuas.

El valor promedio de 2,64 mGy para proyecciones oblicuas es comparable con lo reportado en el documento "National Diagnostic Reference Levels" [11], donde el AGD de mamografía es igual a 2,5 mGy para una muestra de 10 pa- cientes con espesores de mama entre 50-60 $\mathrm{mm}$, para proyecciones oblicuas mediolaterales.

En el estudio de Vanaudenhove et al. [7], para proyecciones cráneo caudales (CC) se obtuvo un valor de AGD igual a $1,73 \mathrm{mGy}$ y $1,90 \mathrm{mGy}$ para proyecciones oblicuas (MLO), utilizando una muestra de más de 39000 pacientes y tomando valores de dosis de 300 equipos de mamografía. Los valores de AGD en el presente estudio son superiores en 0,79 $\mathrm{mGy}$ para proyecciones $\mathrm{CC}$, y $0,74 \mathrm{mGy}$ para proyecciones oblicuas, en comparación con [7].

Los valores de AGD correspondientes al tercer cuartil del diagrama de cajas para mamografía se pueden observar en los correspondientes diagramas de frecuencias de las figuras $6 a, 6 b, 6 c$ y $6 \mathrm{~d}$.

\subsection{Nivel de referencia de dosis en tomografía}

En la figura 7 se muestra el diagrama de cajas para los estudios de tomografía. En la tabla 4 se presentan los valores de $\mathrm{CTDI}_{\text {vol }}$ y DLP definidos como los valores de DRL a partir del tercer cuartil del diagrama de cajas en este estudio. Se presenta también la comparación con los valores de Serra et al. [9], donde los DRL son definidos con la mediana; los valores de Benmessaoud et al. [5] y el NDRL [11], donde los DRL son definidos con el tercer cuartil. En la figura 8 se presentan los diagramas de frecuencias para cráneo, tórax y abdomen para paciente mediano.

Con respecto al DRL de tomografía de cráneo, se obtuvieron valores de dosis inferiores a los de Serra et al. [9], donde los CTDI ${ }_{\text {vol }}$ y DLP fueron 52,4 mGy y 1087,4 $\mathrm{mGy.cm}$, respectivamente para el análisis de la mediana de 73 datos, con $120 \mathrm{kVp}$ en una clasificación por indicación clínica para pacientes mayores de 45 años. En comparación con el estudio de Benmessaoud et al. [5], también se obtuvieron valores de dosis similares, donde los CTDI ${ }_{\text {vol }}$ fueron iguales a $58 \mathrm{mGy}$ para TC de cráneo, y $15 \mathrm{mGy}$ para TC de tórax y abdomen-pelvis. En el mismo estudio, los valores publicados de DLP fueron 1298 y 944 mGy.cm para cráneo y tórax respectivamente, siendo mayores que los alcanzados en el presente estudio. En comparación con los valores del estudio del NDRL [11], los DLP de este trabajo son inferiores para cráneo y abdomen, y mayores para tórax. 
(a)

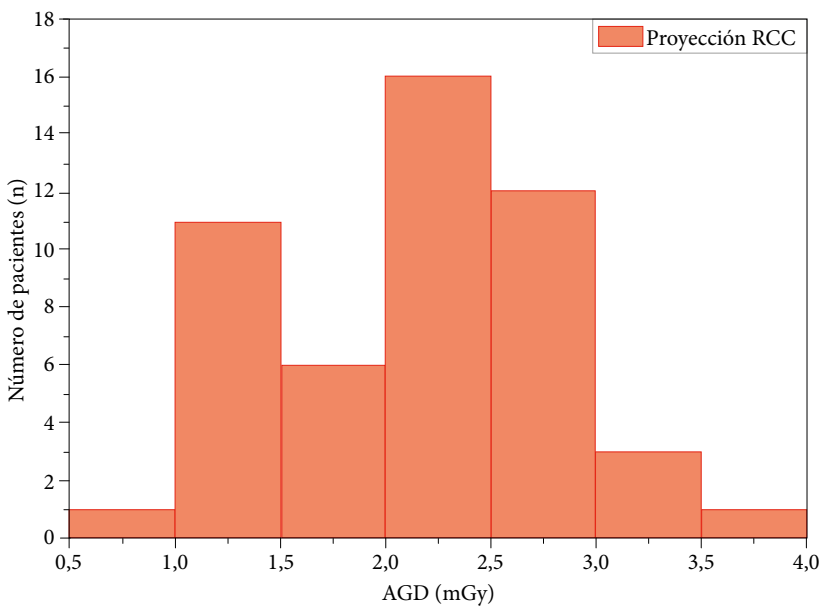

(c)

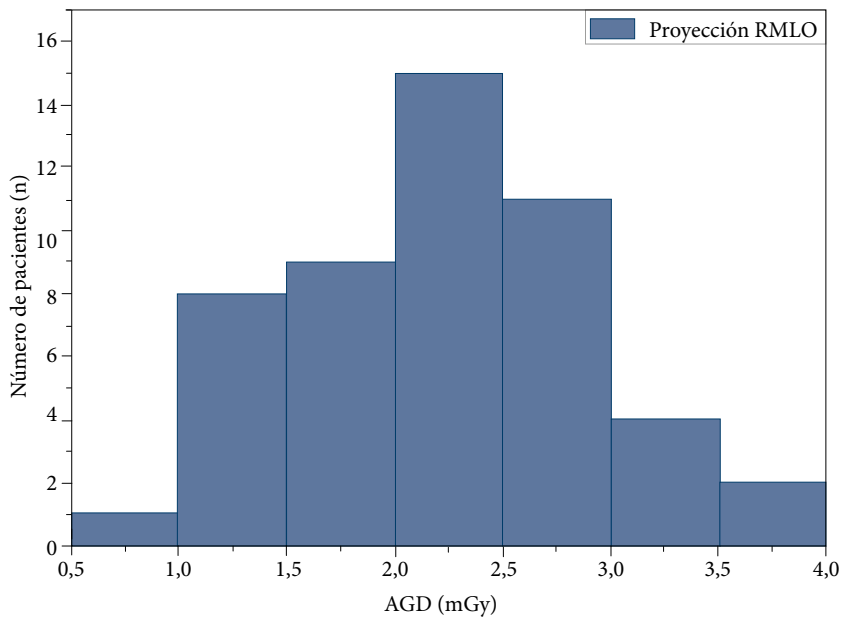

(b)

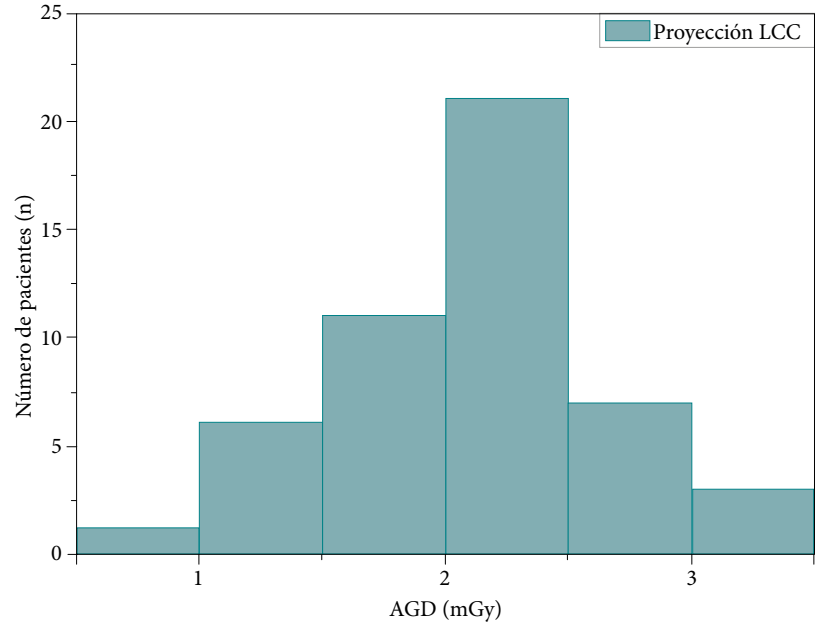

(d)

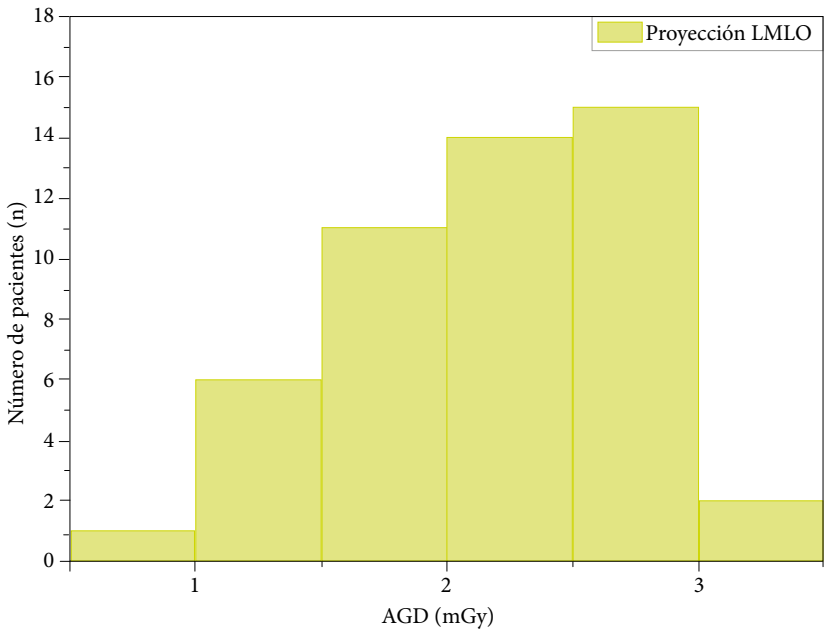

Figura 6. Diagrama de frecuencias del AGD

a) proyección RCC; b) proyección LCC; c) proyección RMLO; d) proyección LMLO.

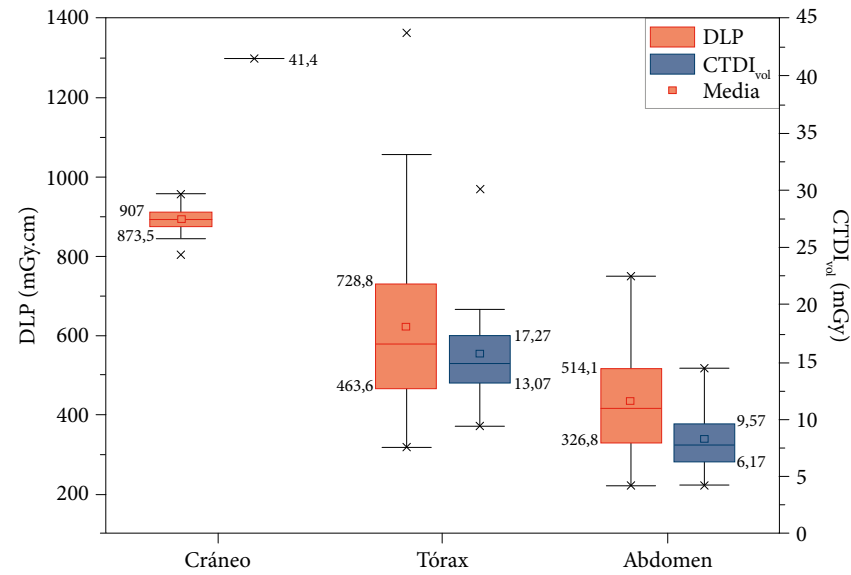

Figura 7. Gráfico de cajas para CTDI ${ }_{\text {vol }}$ (derecha) y DLP (izquierda) para tomografía de cráneo, tórax y abdomen en pacientes adultos medianos 
Tabla 4. DRL para los procedimientos de tomografía de cráneo, tórax y abdomen

\begin{tabular}{|c|c|c|c|c|c|c|c|}
\hline \multirow{2}{*}{ Estudio } & \multicolumn{2}{|c|}{$\begin{array}{c}\text { DRL } \\
\text { Este estudio }\end{array}$} & \multicolumn{2}{|c|}{$\begin{array}{c}\text { DRL } \\
\text { Serra et al. [9] }\end{array}$} & \multicolumn{2}{|c|}{$\begin{array}{c}\text { DRL } \\
\text { Benmessaoud et al. [5] }\end{array}$} & \multirow{2}{*}{$\frac{\text { NDRL [11] }}{\text { DLP (mGy.cm) }}$} \\
\hline & $\mathrm{CTDI}_{\mathrm{vol}}(\mathrm{mGy})$ & DLP (mGy.cm) & $\mathrm{CTDI}_{\mathrm{vol}}(\mathrm{mGy})$ & DLP (mGy.cm) & $\mathrm{CTDI}_{\mathrm{vol}}(\mathrm{mGy})$ & DLP (mGy.cm) & \\
\hline TC cráneo & 41,4 & 907,0 & 52,4 & 1087,4 & 58 & 1298 & 970 \\
\hline TC tórax & 17,27 & 728,8 & ND & ND & 15 & 944 & 610 \\
\hline TC abdomen & 9,57 & 515,1 & ND & ND & ND & ND & 910 \\
\hline
\end{tabular}

ND: no disponible en la publicación.

(a)

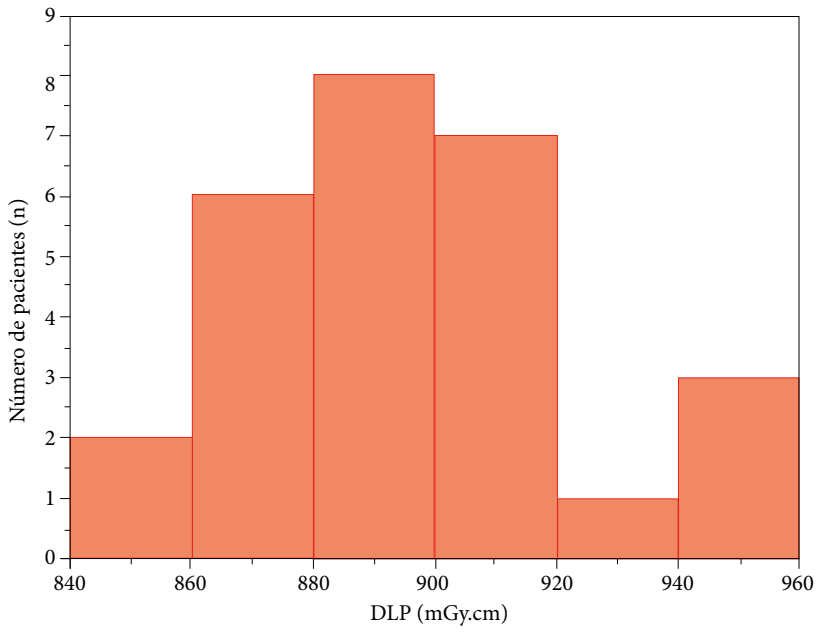

(b)

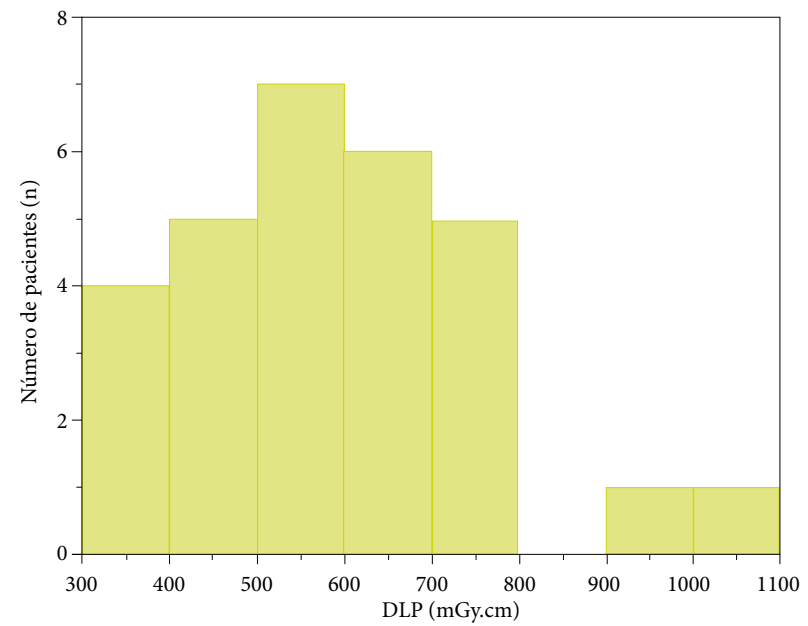

(c)

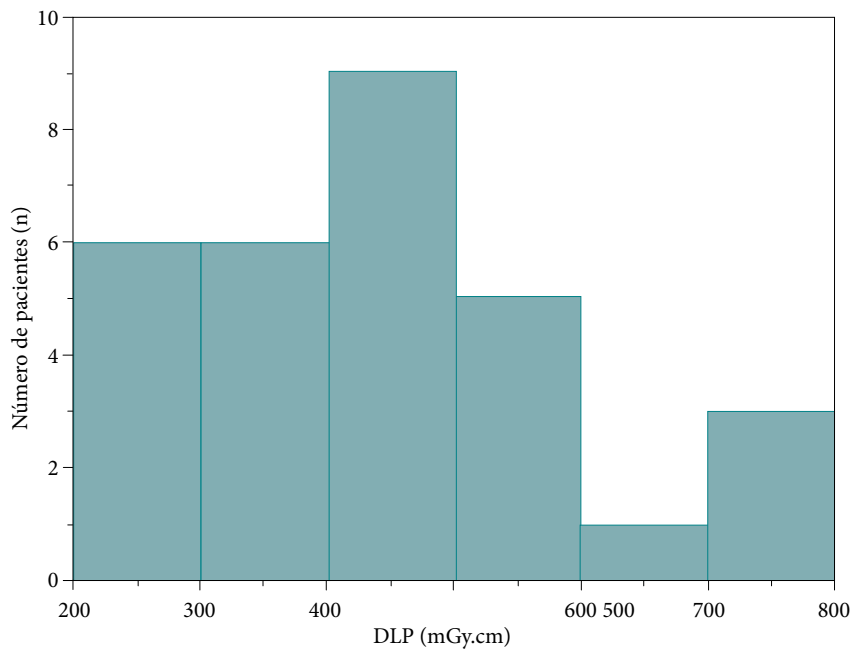

Figura 8. Diagrama de frecuencias del DLP total para paciente mediano a) cráneo; b) tórax; c) abdomen.

\subsection{Niveles de referencia de dosis en procedimientos guiados por fluoroscopia con arco en $\mathrm{C}$}

En la figura 9 se muestra el diagrama de cajas para los estudios guiados por fluoroscopia. En la figura 10 se presentan los diagramas de frecuencias para los mismos estudios. En ambos tipos de diagramas se discriminaron los valores de dosis atípicos.
Los DAP de referencia fueron: 2,68 Gy. $\mathrm{cm}^{2}$ para CPRE; 2,88 Gy.cm² para HSG, y 9,22 Gy.cm² para colon por enema. Los resultados para nuestra institución son muy similares a los DAP presentados en NDRL [11] del Reino Unido, donde los valores publicados son 2,0 Gy. $\mathrm{cm}^{2}$ para HSG. Para el estudio de colon por enema, los valores de dosis en este estudio son inferiores a los reportados en el NDRL [11] con 


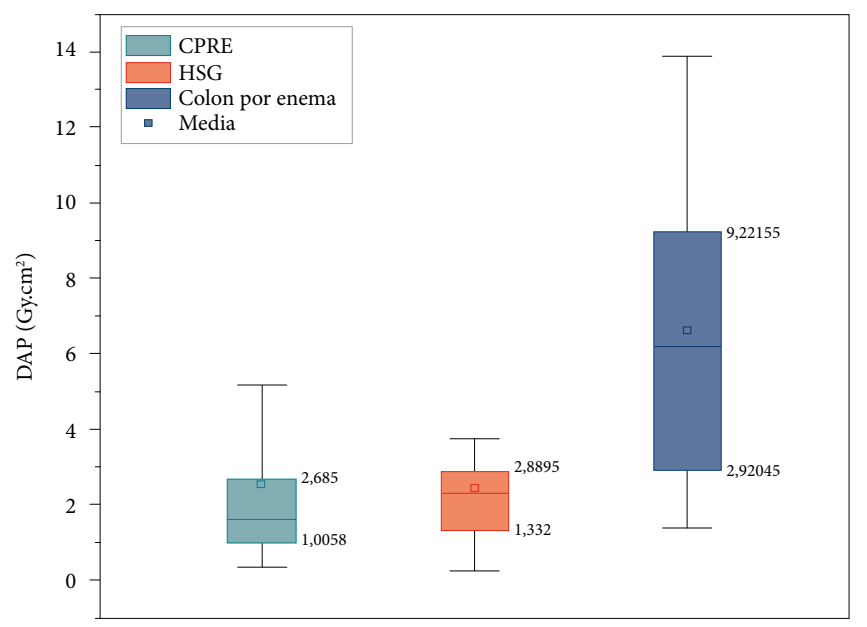

Figura 9. Gráfico de cajas de DAP para procedimientos guiados por fluoroscopia: CPRE, HSG y colon por enema

(a)

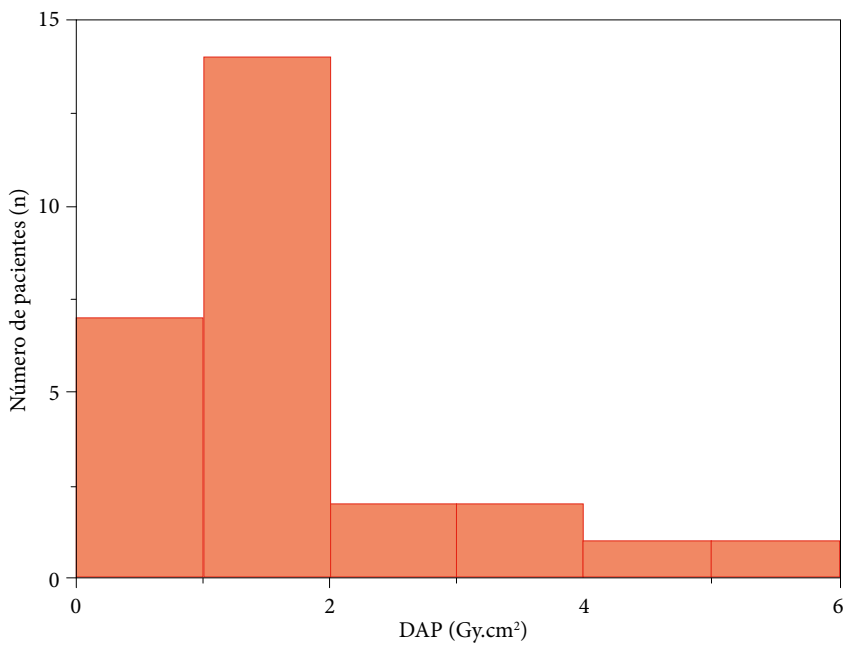

(b)

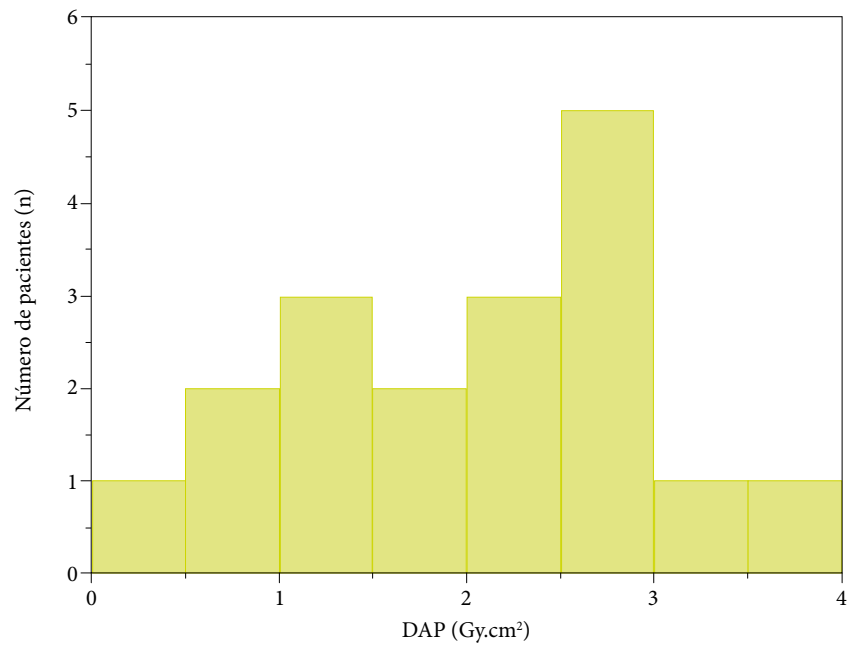

(c)

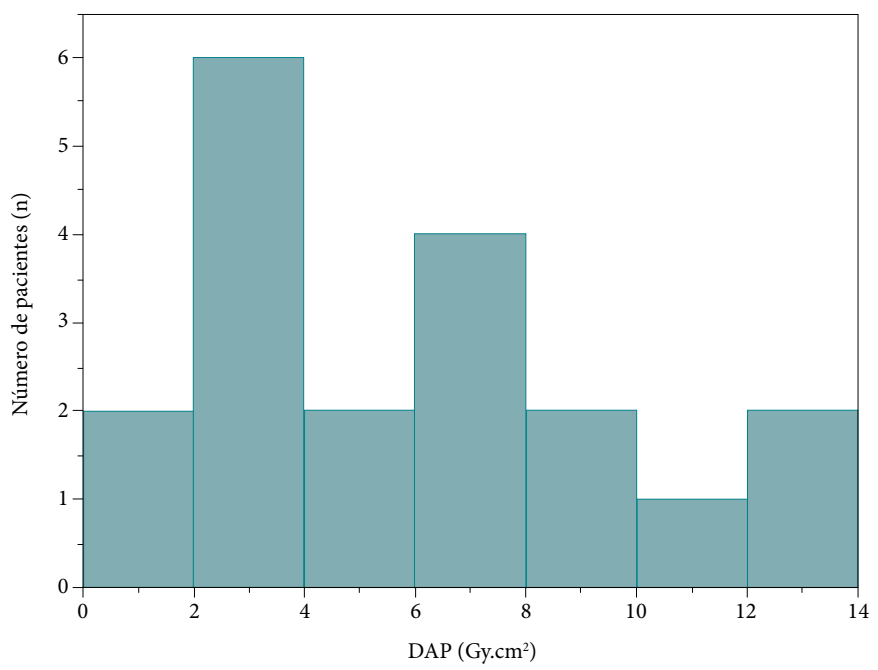

Figura 10. Diagrama de frecuencias del DAP a) CPRE; b) HSP; c) colon por enema. 
valores de hasta $23 \mathrm{~Gy} . \mathrm{cm}^{2}$. Vale la pena mencionar que las dosis estimadas entre procedimientos pueden presentar una dispersión considerable debido a los tiempos de exposición, al espesor del área estudiada del paciente y a la posición del tubo de rayos $\mathrm{X}$ propia de cada estudio.

Los valores de DAP correspondientes al tercer cuartil del diagrama de cajas para estudios guiados por fluoroscopia se pueden observar en los correspondientes diagramas de frecuencias de las figuras $10 \mathrm{a}, 10 \mathrm{~b}$ y $10 \mathrm{c}$.

\section{Conclusiones}

Los niveles de referencia de dosis en procedimientos diagnósticos derivados de este estudio para SES-HUC han arrojado valores comparables a los publicados por otros autores en análisis semejantes. Los DRL obtenidos presentan diferencias según el tamaño del paciente, por lo que es adecuado clasificar por intervalos de peso como lo recomienda la ICRP [2] y como fue realizado en este trabajo. La clasificación de los estudios por técnicas y parámetros de adquisición como el kVp también han permitido una adecuada comparación con algunas publicaciones.

Las comparaciones con estudios de otros autores pueden llegar a ser desafiantes por la diversidad de métodos aplicados en la conformación y clasificación de la muestra de los grupos; principalmente, se observa una gran heterogeneidad en relación con las clasificaciones por peso, tamaño, género y edad, así como en la definición de la estadística aplicada a la hora de definir los DRL por valores de media, mediana, tercer cuartil, entre otros. El seguimiento de protocolos internacionales puede contribuir a la unificación e intercomparación de resultados entre instituciones [7].

Debe tenerse en cuenta que los DRL deberían actualizarse en caso de cambio de equipo o tecnología, o en caso de cambio del tubo de rayos X; igualmente, sería recomendable realizar actualizaciones de los valores de acuerdo con la obsolescencia del equipo de adquisición de imagen o según la recomendación del ente regulador.

Además del cumplimiento de los requisitos para licenciamiento de equipos emisores de radiación establecidos por los entes reguladores, la evaluación de estos niveles puede ser una herramienta útil para la optimización y el seguimiento de las dosis entregadas a los pacientes a nivel institucional. Esta optimización puede permitir la reducción de la dosis recibida por los pacientes, sin afectar la calidad de la imagen diagnóstica o, al contrario, aumentar las dosis entregadas con el fin de mejorarla, para así mantenerse dentro de los niveles de referencia institucionales y cumplir siempre con el principio de ALARA (as low as reasonably achievable: "tan bajo como sea razonablemente posible"). Posterior a este trabajo, realizaremos la validación dosimétrica de los valores estimados en este estudio.

\section{Referencias}

[1] International Atomic Energy Agency (IAEA), "Protección radiológica de los pacientes". [Internet]. Disponible en https://rpop.iaea.org/RPOP/RPoP/Content-es/InformationFor/Patients/information-public/index.htm

[2] International Commission on Radiological Protection (ICRP). (2017). "Diagnostic reference levels in medical imaging. ICRP Publication 135”. Ann. ICRP 46(1). [Internet]. Disponible en https://www.icrp.org/publication. asp?id=icrp\%20publication\%20135.

[3] Ministerio de Salud y Protección Social. "Resolución 482 de 2018". [Internet]. Disponible en https://www. icbf.gov.co/cargues/avance/docs/resolucion_minsaludps_0482_2018.htm.

[4] Z. Vawda, R. Pitcher, J. Akudugu et al., "Diagnostic reference levels for paediatric computed tomography," South African Journal of Radiology, vol. 19, n. ${ }^{\circ} 2$, pp. 10 13, 2015, https://doi.org/10.4102/sajr.v19i2.846.

[5] M. Benmessaoud, A. Housni, M. Elmabrouki et al., "Derivation of local diagnostic reference levels for common adult computed tomography examinations in Moroccan Hospital", Radiation Protection Dosimetry, vol. 194, n. ${ }^{\circ} 4$, pp. 208-213, 2021, https://doi.org/10.1093/ $\mathrm{rpd} / \mathrm{ncab} 095$.

[6] C. J. D'Orsi, E. A. Sickles, E. B. Mendelson et al., ACR BIRADS Atlas, Breast Imaging Reporting and Data System, Virginia: American College of Radiology, 2013.

[7] T. Vanaudenhove, K. V. Slambrouck y A. Fremout, "Niveaux de référence diagnostiques nationaux en radiologie”, Bruselas: Agence Fédérale de Contrôle Nucléaire, 2016.

[8] M. J. Fontelles, Bioestatística: aplicada à pesquisa experimental, vol. 1, São Paulo: Livraria da Física, 2012.

[9] A. Serra, C. Ramírez, J. Véliz et al., "Valores típicos de dosis para tomografía computada de cerebro en paci- 
entes adultos", Revista Chilena de Radiología, vol. 26, n. ${ }^{\circ}$ 1, pp. 25-31, 2020, https://doi.org/10.4067/s071793082020000100025.

[10]A. Almén, J. Guðjónsdóttir, N. Heimland et al., "Establishing paediatric diagnostic reference levels using reference curves - A feasibility study including conventional and CT examinations", Physica Medica, vol.
87, n. ${ }^{\circ}$ 5, pp. 65-72, 2021, https://doi.org/10.1016/j. ejmp.2021.05.035.

[11] "National Diagnostic Reference Levels (NDRLs) from 19 August 2019”. [Internet]. UK Government, 2021. Disponible en https://www.gov.uk/government/publications/diagnostic-radiology-national-diagnostic-reference-levels-ndrls/ndrl. 